\title{
HIGH RESOLUTION IMAGE RECONSTRUCTION AT YUNNAN OBSERVATORY*
}

\author{
P.Qiı, Y.Qiu, P.Qian, Z.Liu \\ (Yunnan, Observatory, Kunming 650011, China, P.R.)
}

The research on the high resolution image reconstruction has been carried out at the Yunnan Observatory, the Chinese Academy of Sciences, since 1983. At the early stage in 1983-1987, our research was concentrated on the Speckle Interferometry[1]. The first developed speckle camera for using the film as the recording medium was made up of an intensifier with a gain of 57,000 and an SB-408-B oscilloscope camera. In May, 1985, 144 speckle interferograms of the binary $\zeta B$ oo and the unresolved single star 32 Boo apiece were obtained with the speckle camera attached to the 1-M Rcc telescope, and the average power spectrum of $\zeta B O O$ was then acquired by means of the Optical Fourier Transformation[2]. Soon afterwords, the optical couple of the intensifier to a $\operatorname{RCA~} \operatorname{CCD}(320 \times 512$ pixels $)$ through a lens was used to replace the film and the oscilloscope camera, thereby making an improvement on the detectability up to $\tau^{m}$ from $4^{\prime \prime \prime}$. In December, 1986 the improved camera attached to 1-M telescope was used to observe three binary stars, i.e. Kui23, $\sum 73$ and $\sum 346.4 B$. The speckle interferograms were processed with a computer and angular seperations of these binaries were obtained approximate to the diffraction-limited resolution of the telescope[3].

From 1988 on, our attention has been turned to the 2-D high resolution image reconstruction. After careful analysis of the available various methods for the image reconstruction, the Speckle Masking[4-7] is defined as the main target of our research and experiment. The reason is as follow: The Speckle Masking is based on the comparatively perfect and strict theory, containing much more high frequency information by adopting estimate of the third-order statistical moment[8], effectively depressing the noise by means of the miltiway average in phase and modulus recursion and having the advantages, such as the insensitivity to the additive noise and telescope aberration and the universal application to astronomical objects

After the completion of the 4-D processing scheme and computer programing for the bispectra, the computer simulation and preliminarily astronomical test were successively carried out. For the computer simulation, the true atmosphere-telescope model was adopted with the purpose of the understanding of the influence of the statistical characteristics of the atmosphere on the rightness and signal-to-noise ratio of the re-

*This subject is supported by National Natural Science loumclation Committee of ('hina. 
stored image, thereby having a good command of the required performance of the image detector, the necessary observation conditions and the priciple of the data processing. The simulation under the true atmosphere was simply recording the sequences of the speckle interferograms $\left\{P_{n}(\vec{x})\right\}_{n=1}^{N}$ of an unresolvable star using the speckle camera on $1-M$ telescope, then a quadruple star $O(\vec{x})=\sum_{m=1}^{4} a_{m} \delta\left(\vec{x}-\vec{x}_{m}\right)$ was made artificially in the computer, with each component having a minimal angular seperation corresponding to 0 ".4. The simulation was divided into two groups. For the first group, the odd sequence $\left\{P_{i}(\vec{x})\right\}_{i},(i=1,3,5, \ldots)$ was convoluted with the man-made object $O(\vec{x})$ to obtain the sequence of the speckle interferograms of the object image, i.e.

$$
I_{i}(\vec{x})=O(\vec{x}) * P_{i}(\vec{x})
$$

And then the bispectrum

$$
<\tilde{I}_{i}^{(3)}(\vec{u}, \vec{v})>=\dot{O}^{(3)}(\vec{u}, \vec{v})<\tilde{P}_{i}^{(3)}(\vec{u}, \vec{v})>
$$

Was calculated.

The even squence $\left\{P_{j}(\vec{x}\}_{j}(j=2,4,6, \ldots)\right.$ was used to calculate the Speckle Masking transfer function $\left\langle\hat{P}_{j}^{(3)}(\vec{u}, \vec{v})>\right.$ and after the deconvolution one may obtain

$$
\dot{O}^{(3)}(\vec{u}, \vec{v})=\dot{O}^{(3)}(\vec{u}, \vec{v})<\tilde{P}_{i}^{(3)}(\vec{u}, \vec{v})>/<\tilde{P}_{j}^{(3)}(\vec{u}, \vec{v})>
$$

Finally, from $\dot{O}^{(3)}(\vec{u}, \vec{v})$, the approximate spectrum of the object $\tilde{O}^{\prime}(\vec{u})$ was derived.

For the second group, $\left\{P_{i}(\vec{x})\right\}_{i}$ and $\left\{P_{j}(\vec{x})\right\}_{j}$ were acquired from the same star or different stars at different time, and after the same processing as that of the first group, the another spectrum of the object $\dot{O}^{\prime \prime}(\vec{u})$ can also be derived.

It is shown from the comparision of the $\tilde{O}^{\prime}$ or $\hat{O}^{\prime \prime}$ with $\tilde{O}$ that the relationship of the mutual positions of the substars in $\dot{O}^{\prime}$ and $\dot{O}^{\prime \prime}$ is quite accurate (with the error $\leq 0^{\prime \prime} .1$ ). However, the distribution of the brightnesses of the substars shows a greater error, especially those of $\dot{O}^{\prime \prime}[9]$.

In September, 1990, the speckle observation of two binaries, $\zeta S g r$ and $\eta O_{p h}$, and a single star $\delta C^{\prime}\left(a_{l}\right)$ were performed. 120 speckle interferograms of each object were processed for the preliminary test of the astronomical image reconstruction. The results were obviously worse than those from the simulation. However, both results have the same characteristics, i.e.the comparatively accurate relationship of the positions and the greater error of the distribution of the brightnesses[10]. This can be explained as follows: as far as the Speckle Masking is concerned, the transfer function in the observation of the object should be identical with that of the single star as far as possible, i.e.

$$
\left\langle\tilde{P}_{i}^{(3)}(\vec{u}, \vec{v})>\cong<\hat{P}_{j}^{(3)}(\vec{u}, \vec{v})>\right.
$$

And both should have the minimal variance so as to make the restoration of the phase and modulus of the object more accurate. Substantially, this involves the problem of 
the ergodicity of the third-order moment in the random process $P(\vec{x})$. In practice, the ergodicity of the third-order moment can only be approximately tenable if the number $\mathrm{N}$ of adequate samples, both object and single star, is obtained in the shortest possible interval of time and space during the observation. Accordingly, the detector should have the performance of higher sensitivity, lower noise and rapid readout. Since the detectability of our speckle camera is only about $\tau^{m}$, the noises in the tube and $C C D$ are greater and the readout time for each frame is 5 seconds, then it should take 2 hours(including the time for some auxiliary work)at least to obtain 300 speckle interferograms of the object and single star, which still falls far short of the requirement of the ergodicity and the number $\mathrm{N}$ of samples is too small, thereby resulting in a bad signal-to- noise ratio. Even so, since the Speckle Masking transfer function is real, $<\dot{P}_{j}^{(3)}(\vec{u}, \vec{r})>$ does not effect the restored phase, but does effect the modulus. This is the reason why the restored positions are more accurate than brightnesses.

Therefore, the whole thing we have done has so far been the experimental one only. The key to applying the Speckle Masking to the high resolution observation of stars lies in the solution of the problem of an image detector. Now, a 2-D photon-counting camera is under development.

References

1. Labeyrie,A., Astron.Astrophys., 6, 85(1970).

2. Wang, Y., et.al., Publications of Yunnan Obs., 2, 51(1986).

3. Wang,Y., et.al., Acta Astronomical Sinica, 4, 410(1987).

4. Weigelt,G., Opt.commun., 21, 55(1977).

5. Weigelt,G., Wirnitzer,B., Opt.Lett., 8, 389(1983).

6. Lohmann,A.W., Weigelt,G.P. and Wirnitzer,B., Appl.Opt., 22, 4028(1983).

7. Bartelt,H., Lohmann,A.W. and Wirnitzer,B., Appl.Opt., 23, 3121(1984).

8. Qiu,P., Progress in Astronomy, 4, 328(1989).

9. Qiu,Y., et.al., Publications of Yunnan Obs., 2, 42(1992).

10.Qiu,Y., et.al., Acta Astronomical Sinica, 3, 280(1992). 